\title{
Iris eyelid detection based on adaptive sectional line fitting
}

\author{
Ji Xiaocun ${ }^{1, a^{*}}$, Chen Houjin ${ }^{1, b}$, Li Jupeng ${ }^{1, c}$
}

${ }^{1}$ School of Electronic \& Information Engineering, Beijing Jiaotong University, Beijing, China

a13120017@bjtu.edu.cn, bhjchen@bjtu.edu.cn, Clijupeng@bjtu.edu.cn

Key Words: Iris recognition, Eyelid detection, Sectional straight line, Mathematical morphology

Abstract. It is the premise condition of iris recognition that we segment iris image effectively. Most of the iris images are interfered by eyelid, so it is important for iris image segmentation that detecting eyelid accurately. An iris eyelid detection method based on adaptive sectional line fitting was presented in this paper. The knowledge about edge detection and mathematical morphology is applicable to detect the eyelid area adaptively. The result shows our method can detect the corresponding eyelid area accurately and effectively compared to traditional eyelid detection methods.

\section{Introduction}

Iris recognition has obtained widely attention because of its unique biological characteristics in recent years. Many sample iris images have a great quantity of noise influence, it will seriously affect the feature extraction and recognition rate if we don't remove them from the iris image. At present, it has three main methods to detect the eyelid: 1) Curve positioning based on the parabola ${ }^{[1]}$; 2) Circular ring positioning ${ }^{[2]}$; 3) Single straight line positioning ${ }^{[3]}$. The first model has high precision and large amount of calculation. It is difficult to meet the requirements of real-time. The second model has the fastest positioning speed but the lowest location accuracy. The third model has improved the formal methods. But it will lead to the wrong outcome when the upper or lower eyelid is close to pupil ${ }^{[4]}$.

According to the limitations of above three kinds of methods, this paper proposes an improved detection method based on adaptive sectional straight line fitting. We determine whether to do sectional line detection by computing the distance between the single positioning line and the pupil. The method ensures the accuracy and improves the positioning time with morphology processing and edge detection.

\section{Eyelid detection algorithms}

The iris eyelid image consists of upper eyelid and lower eyelid. In consideration of the similarity of detecting method, we only detect the upper eyelid in this paper. The overall flow chart of iris eyelid detection is shown in figure 1:

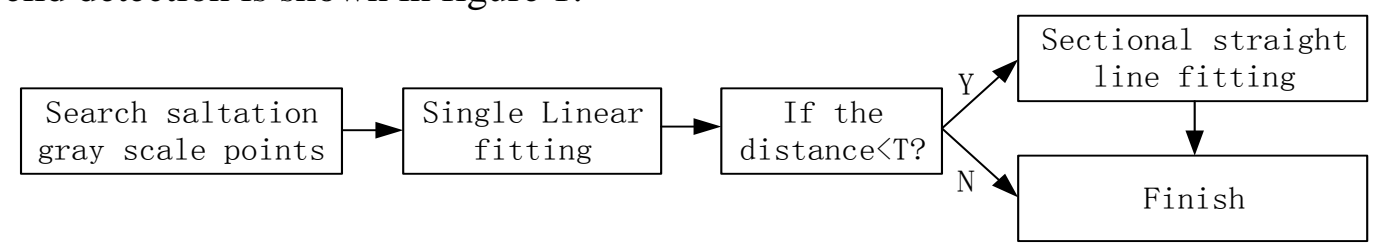

Figure 1 Flow chart of iris eyelid detection

\subsection{Search saltation gray scale points}

Due to the saltation gray scale points are usually on the focus of eyelid and outer edge, it is feasible to detect the eyelid area on the basis of outer edge positioning accurately. We pick up a set of pixels $\left\{A_{1}, A_{2}, A_{3} \ldots A_{360}\right\}$ from the outer edge. The coordinate of each $A i(i \in(1,360))$ is:

$$
\begin{gathered}
x_{i}=w_{x}+w_{r} \times \cos (\theta(i)) . \\
y_{i}=w_{Y}-w_{r} \times \sin (\theta(i)) . \\
\theta(i)=\frac{\pi}{180} i, i \in(1,2,3 \ldots 360) .
\end{gathered}
$$

Where $A_{i}=\left(x_{i}, y_{i}\right),\left(W_{X}, W_{Y}\right)$ is the central coordinate of outer edge, $W_{r}$ is the radius of outer edge.

Then these points on the outer edge will be divided into four regions as in figure 2(a). We can 
search the edge points of upper eyelid in region 1 and region 2 by formula 4 and 5 .

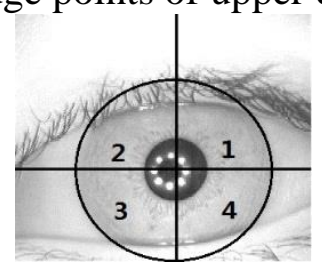

(a) Segmentation area

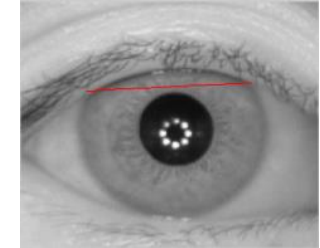

(b) Single line detection

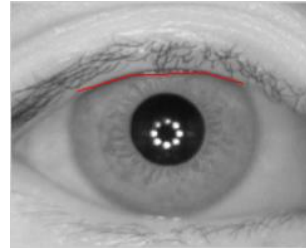

(c) Sectional line detection

Figure 2 Single and sectional line detection

$$
\begin{aligned}
& \left|\sum_{i}^{i+4} f\left(A_{i}\right)-\sum_{i}^{i-4} f\left(A_{i}\right)\right|>\operatorname{Gray}(5 \leq i \leq 86) . \\
& \left|\sum_{i}^{i+4} f\left(A_{i}\right)-\sum_{i}^{i-4} f\left(A_{i}\right)\right|>\operatorname{Gray}(95 \leq i \leq 176) .
\end{aligned}
$$

In consideration of both sides of pupil areas usually exist little eyelashes compared with the upper eyelid areas, we search these points from both sides of the pupil to upper and lower eyelids. If we can find the first point that the difference of gray sum between left sides and right sides is greater than a threshold ${ }^{[5]}$, we should register it and regard it as the focus of eyelid and outer edge. If the point cannot be found, the outer edge is not sheltered from eyelid.

\subsection{Single linear fitting}

If we can find the focus of eyelid and outer edge: HLedge and HRedge, we will draw a straight line to locate the eyelid area by the following formula:

$$
k\left(X-x_{2}\right)=\left(Y-y_{2}\right)
$$

where $\left(x_{1}, y_{1}\right)$ is the coordinate of HLedge, $\left(x_{2}, y_{2}\right)$ is the coordinate of HRedge, $k=\left(y_{2}-y_{1}\right) /\left(x_{2}-x_{1}\right)$. Then we can judge the distance between this straight line and the pupil by formula (7) and (8).

$$
\text { distance }=\frac{\left|A \times W_{X}+B \times W_{Y}+C\right|}{\sqrt{A^{2}+B^{2}}} \text {. }
$$

Where $A=k, B=-1, C=y_{2}-k x_{2}, N_{r}$ is the radius of inner edge. If distance $<N_{r}+\mathrm{T}$, we need to do sectional straight line fitting. If distance $>=N_{r}+\mathrm{T}$, we don't need. $\mathrm{T}$ is based on prior knowledge, we set it to 15 so that we can keep the iris information as much as possible.

\subsection{Sectional line detection}

If we adopt single line to detect the eyelid, the located line may cut the pupil or close to the pupil as in figure 2(b). It will miss a lot of key information in following feature extraction and coding so that we cannot recognize accurately. Given all that, in order to avoid these disadvantages, we adopt adaptive sectional line to detect eyelid as in figure 2(c).

The following is the algorithms of sectional straight line detection:

1) According to the positioning result of inner and outer edge, we determine the upper eyelid area and cut it out by formula 9 and 10 so that we can reduce the search region.

$$
\begin{gathered}
\text { Heyelidedge }(i, j)=I(i, j)\left(1 \leq i \leq x_{\text {pupil }}-r, 1 \leq j \leq N\right) . \\
\text { Leyelidedge }\left(i-\left(x_{\text {pupil }}+r\right), j\right)=I(i, j)\left(x_{\text {pupil }}+r+1 \leq i \leq M, 1 \leq j \leq N\right) .
\end{gathered}
$$


Where $X_{\text {pupil }}$ is the row of the center of inner edge; $r$ is the radius of inner edge; $M, N$ are the width and height of iris image.

2) In order to reduce the effect of the eyelashes on the edge of eyelid, we adopt horizontal edge detection. The edge detection template is shown in Figure 3(a).

$$
\left[\begin{array}{rrr}
-1 & -2 & -3 \\
0 & 0 & 0 \\
1 & 2 & 3
\end{array}\right]
$$

(a) Edge detection template

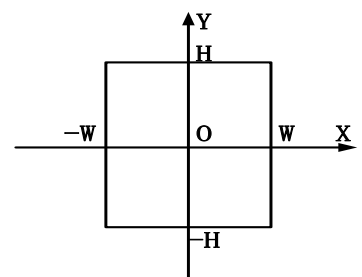

(b) Rectangular detection template

Figure 3 Detection template

3) In consideration of the influence of local noise on edge detection, we do some processing, such as mathematic morphology, connected domain detection and region filling. As the figure 3(b) shows that, we design a rectangular detection template ${ }^{[6]}$ to detect edge strength value. It has stronger robustness than search directly. The template is divided into two parts by $\mathrm{x}$-axis. The direction of y-axis is regarded as template direction and search direction.

We search for two of the upper eyelid edge points from bottom to up along inner edge's left and right boundary. When searching for the left and right edge points, we calculate the difference of grey sum between up parts and down parts along searching path by the following formula.

$$
G(x, y)=\sum_{j=-W}^{W} \sum_{i=1}^{H} I((x+i),(y+j))-\sum_{j=-W}^{W} \sum_{i=1}^{H} I((x-i),(y+j)) \text {. }
$$

Left and right edge points are:

$$
\begin{aligned}
& P_{L}\left(x_{L}, y_{L}\right)=\arg \max _{a \leq x \leq b, y=W_{y}-r}\{G(x, y)\} . \\
& P_{R}\left(x_{R}, y_{R}\right)=\arg \max _{a \leq x \leq b, y=W_{y}+r}\{G(x, y)\} .
\end{aligned}
$$

where $a=W_{x}-\sqrt{W_{r}^{2}-\left(y-W_{y}\right)^{2}}, b=W_{r}-r$.

Although this method can eliminate the vast majority of the effects of local noise, some extreme cases also exist. We may find many edge points or we cannot find such points. In the first case, we can judge these points whether are in the largest connected domain. If it is not in, we give it up. In the second case, we can adjust the searching area by shifting the two boundaries to central with a tiny distance and continue to search ${ }^{[7]}$.

4) After above, we can find four points including $\operatorname{HLedge}\left(x_{1}, y_{1}\right), P_{L}\left(x_{L}, y_{L}\right), P_{R}\left(x_{R}, y_{R}\right)$ and $\operatorname{HRedge}\left(x_{2}, y_{2}\right)$. Then we can solve the linear equation by formula 14 .

$$
\left\{\begin{array}{l}
L 1: \frac{y_{L}-y_{1}}{x_{L}-x_{1}}\left(x-x_{1}\right)=\left(y-y_{1}\right) . \\
L 2: \frac{y_{R}-y_{L}}{x_{R}-x_{L}}\left(x-x_{L}\right)=\left(y-y_{L}\right) \\
L 3: \frac{y_{2}-y_{R}}{x_{L}-x_{R}}\left(x-x_{R}\right)=\left(y-y_{R}\right) .
\end{array}\right.
$$




\section{Experimental results and analysis}

\subsection{The results of sectional linear positioning eyelids}

The detection algorithm in this paper is practiced and simulated under the environment of Matlab 7.10. Iris images used in the experiment are from CISIA1.0 ${ }^{[8]}$ iris library (contains 1307 images) of Chinese academy of sciences. The size of each image is $320 x 280$. Figure 4 shows that the process of adaptive sectional line fitting.

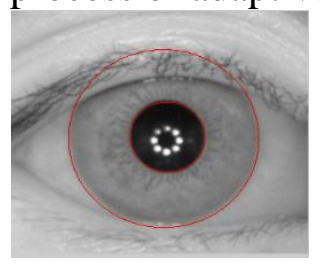

(a) Inner and outer edge position
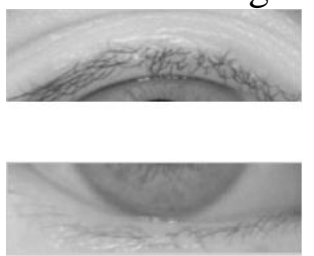

(b) Upper and lower eyelid image
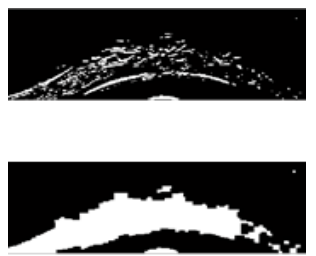

(c) Morphological processing

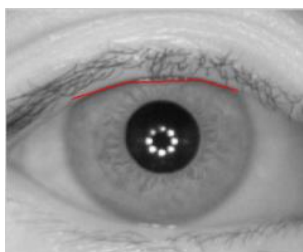

(d) Upper eyelid position Figure 4 Process of eyelid detection

\subsection{Results analysis}

In order to validate the performance of this method, we have compared with the existing contrast detection algorithm.

Table 1 Upper eyelid detection

\begin{tabular}{ccc}
\hline Algorithm & Average time[s] & Accuracy[\%] \\
\hline Parabola model & 4.323 & 95.72 \\
Circular ring & 0.912 & 88.12 \\
Single line & 1.126 & 90.13 \\
This paper & 1.174 & 93.68 \\
\hline & Table 2 Lower eyelid detection & \\
\hline Algorithm & Average time[s] & Accuracy[\%] \\
\hline Parabola model & 4.014 & 93.12 \\
Circular ring & 0.883 & 86.64 \\
Single line & 1.014 & 89.77 \\
This paper & 1.102 & 94.78 \\
\hline
\end{tabular}

From table 1 and 2, it shows that we can detect the eyelid region on the iris image accurately by adaptive sectional line fitting. On the one hand, it owes to the accurate outer edge. On the other hand, it benefits from morphological image processing. It does not need a lot of points to iterative computations compared with the parabola model. At the same time, it improves the single line model and has stronger robustness than other methods.

\section{Summary}

In this paper, an efficient and adaptive eyelid detection method is presented. Three sectional positioning lines are used to detect the eyelid area. Eyelid edge points are found by edge detection and morphological processing. Whether to do sectional line fitting is determined by the distance between the pupil and the primary positioning line. This method not only ensures the detection speed but also guarantees the detection accuracy. In a word, this method has great significance for evaluating iris image quality and subsequent recognition processing. 


\section{Acknowledgement}

This paper is supported by Beijing Municipal Science and Technology Project (D15110000041 5002), Specialized Research Fund for the Doctoral Program of Higher Education (20120009120001 ), and Fundamental Research Funds for the Central Universities (2015JBM015).

\section{References}

[1] Ghassan J.Mohammed. A New Localization Method for Iris Recognition Based on Angular Integral Projection Function, 2009, 10(2): 316-320

[2] Daugman J. How iris recognition works [J]. IEEE Transactions on Circuit and System for Video Technology, 2004, 14(1):21-30.

[3] J Daugman. High confidential visual recognition by test of statistical independence[J]. IEEE Trans on pattern analysis and machine intelligence, 1993,15(11): 1148-1161.

[4] Wildes R. Iris recognition: an emerging biometric technology[J]. Proceedings of the IEEE, 1997, 85(9): 1348-1363.

[5] Wai- Kin Kong. Detecting eyelash and reflection for accurate iris segmentation [J]. International Journal of Pattern Recognition and Artificial Intelligence, 2003, 17(6): 1025-1034.

[6] Guang Zhuxu, Zhang Zaifeng, Ma Yide. Local Intensity Var-iation Analysis for Iris Recognition. Pattern Recogn-ition, 2004, 37 (6): 1287-1298.

[7] N.P Joshi, C.P. Shah. A novel approach implementation of eyelid detection in biometric applications. Nirma university international conference on engineering, 2012, 6(8):2-8.

[8] CASIA Iris Image Database V-1.0, http://www.cbsr.ia.ac.cn/IrisDatabase.htm, 2013. 